\title{
Constructive Interference Based Secure Precoding
}

\author{
Muhammad R. A. Khandaker, Christos Masouros and Kai-Kit Wong \\ Department of Electronic and Electrical Engineering \\ University College London \\ Gower Street, London, WC1E 7JE, United Kingdom \\ e-mail: $\{$ m.khandaker, c.masouros, kai-kit.wong\}@ucl.ac.uk
}

\begin{abstract}
Recent advances in interference exploitation showed that exploiting knowledge of interference constructively can improve the receive signal-to-interference and noise ratio (SINR) at the destination. This paper exploits this concept to design artificial noise (AN) beamformers constructive to the intended receiver (IR) yet keeping AN disruptive to possible eavesdroppers (Eves). A multiple-input single-output (MISO) wiretap channel with multiple eavesdroppers scenario has been investigated taking both perfect and imperfect channel information into consideration. The main objective is to improve the receive SINR at the IR through exploitation of AN power in an attempt to minimize the total transmit power, while confusing the Eves.
\end{abstract}

\section{INTRODUCTION}

While dealing with the catastrophe of interference, traditional goal is to suppress or even kill the interference power in order to improve system performance [1]. However, recent developments in interference exploitation techniques have revolutionised this traditional way of managing known interferences [2]. Constrictive interference (CI) precoding approaches suggest that interference power can even contribute to the received signal power if properly exploited [2]-[4]. In particular, downlink beamforming design can be significantly improved by symbol-level precoding of known interferences [5].

Recently, physical-layer security (PLS) approaches have attracted a great deal of attention in the information-theoretic society since the accompanying techniques can afford an extra security layer on top of the traditional cryptographic approaches [6]-[9]. To make physical-layer secrecy viable, we usually need the legitimate user's channel condition to be better than the eavesdroppers'. However, this may not always be guaranteed in practice. To alleviate the dependence on the channel conditions, recent studies showed that the spatial degrees of freedom (DoF) provided by multi-antenna technology can be exploited to degrade the reception of the eavesdroppers [6], [7]. A more operational approach is to send artificially generated noise signals to interfere the eavesdroppers deliberately [6]-[8]. Depending on the extent of eavesdroppers CSI available at the transmitter, different strategies can be applied to generate the optimal AN beams. If no eavesdroppers' CSI is available, then a popular design is the isotropic AN [6], where the message is transmitted in the direction of the intended receiver's channel, and spatiotemporal AN is uniformly spread on the orthogonal subspace of the legitimate channel. On the other hand, with knowledge of the eavesdroppers' CSI to some extent, one can block the eavesdroppers' interception more efficiently by generating spatially selective AN [7], [8]. More recently, an antenna array based directional modulation scheme (DM) has been studied which enhances security through adjusting the amplitude and phase of the transmit signal along a specific direction by varying the length of the reflector antennas for each symbol while scrambling the symbols in other directions [10], [11].

In this paper, we exploit the knowledge of interference available at the transmitter for improving security in wireless systems. In this context, we redesign AN signals in the form of constructive interference to the intended receiver (IR) while keeping AN disruptive to potential eavesdroppers (Eves). We consider a multiple-input single-output (MISO) downlink system in the presence of multiple Eves. We aim at minimizing the total transmit power while boosting the received SINR at the IR as well as degrading the Eves' SINR in an attempt to keep the same below certain threshold. The benefits of constructive interference-based AN precoding scheme is twofold compared to conventional AN-based physical-layer security schemes considered in [6]-[8]. Firstly, the constructive AN will boost the receive SINR at the IR as opposed to the conventional AN-based schemes which attempt to suppress AN signals along the direction of the IR. Secondly, to achieve a predefined level of SINR at the IR, constructive interference based precoding scheme requires lower power compared to conventional AN precoding, thus diminishing inter-user as well as inter-cell interferences. Both perfect and imperfect CSI cases have been investigated. Numerical simulations demonstrate that the proposed constructive AN precoding approach yields superior performance over conventional schemes in terms of transmit power.

\section{System Model}

We consider a MISO downlink system where the transmitter (BS) equipped with $N_{\mathrm{T}}$ transmit antennas intend to transmit a secret message to the IR in the presence of $K$ possible eavesdroppers. The IR and the Eves are all equipped with a single antenna. In order to confuse the Eves, the BS injects AN signals into the secret message in an attempt to reduce the receive SINRs at the Eves. Thus the received signal at the IR and those at the Eves are given, respectively, by $y_{\mathrm{d}}$ and $y_{\mathrm{e}, k}$ :

$$
\begin{aligned}
y_{\mathrm{d}} & =\mathbf{h}_{\mathrm{d}}^{T} \mathbf{x}+n_{\mathrm{d}}, \\
y_{\mathrm{e}, k} & =\mathbf{h}_{\mathrm{e}, k}^{T} \mathbf{x}+n_{\mathrm{e}, k}, \text { for } k=1, \ldots, K,
\end{aligned}
$$


where $\mathbf{h}_{\mathrm{d}}$ and $\mathbf{h}_{\mathrm{e}, k}$ are the complex channel vectors between the BS and the IR and between the BS and the $k$ th Eve, respectively, $n_{\mathrm{d}} \sim \mathcal{C N}\left(0, \sigma_{\mathrm{d}}^{2}\right)$ and $n_{\mathrm{e}, k} \sim \mathcal{C N}\left(0, \sigma_{\mathrm{e}}^{2}\right)$ are the additive Gaussian noises at the IR and the $k$ th Eve, respectively. The BS chooses $\mathbf{x}$ as the sum of information beamforming vector $\mathbf{b}_{\mathrm{d}} s_{\mathrm{d}}$ and the AN vector $\mathbf{b}_{\mathrm{n}} \triangleq \sum_{i=1}^{N} \mathbf{b}_{\mathrm{n}, i} s_{\mathrm{n}, i}$ such that the baseband transmit signal vector is

$$
\mathbf{x}=\mathbf{b}_{\mathrm{d}} s_{\mathrm{d}}+\sum_{i=1}^{N} \mathbf{b}_{\mathrm{n}, i} s_{\mathrm{n}, i},
$$

where $s_{\mathrm{d}} \sim \mathcal{C N}(0,1)$ is the confidential information-bearing symbol for the IR and $s_{\mathrm{n}, i} \sim \mathcal{C N}(0,1), \forall i$, are the AN symbols in which $N$ denotes the number of AN symbols.

Accordingly, the received SINR at the IR is given by

$$
\gamma_{\mathrm{d}}=\frac{\left|\mathbf{h}_{\mathrm{d}}^{T} \mathbf{b}_{\mathrm{d}}\right|^{2}}{\sum_{i=1}^{N}\left|\mathbf{h}_{\mathrm{d}}^{T} \mathbf{b}_{\mathrm{n}, i}\right|^{2}+\sigma_{\mathrm{d}}^{2}},
$$

and that at the $k$ th Eve is given by

$$
\gamma_{\mathrm{e}, k}=\frac{\left|\mathbf{h}_{\mathrm{e}, k}^{T} \mathbf{b}_{\mathrm{d}}\right|^{2}}{\sum_{i=1}^{N}\left|\mathbf{h}_{\mathrm{e}, k}^{T} \mathbf{b}_{\mathrm{n}, i}\right|^{2}+\sigma_{\mathrm{e}}^{2}}, \forall k .
$$

The transmit signal $\mathbf{x}$ can also be expressed as

$$
\mathbf{x}=\mathbf{b}_{\mathrm{d}} s_{\mathrm{d}}+\sum_{i=1}^{N} \mathbf{b}_{\mathrm{n}, i} e^{j\left(\phi_{\mathrm{n}, i}-\phi_{\mathrm{d}}\right)} s_{\mathrm{d}},
$$

where $s_{\mathrm{d}}=d e^{j \phi_{\mathrm{d}}}$. Assuming constant envelop $d=1$, the instantaneous transmit power is given by

$$
P_{\mathrm{T}}=\left\|\mathbf{b}_{\mathrm{d}}+\sum_{i=1}^{N} \mathbf{b}_{\mathrm{n}, i} e^{j\left(\phi_{\mathrm{n}, i}-\phi_{\mathrm{d}}\right)}\right\|^{2} .
$$

\section{PROBlem Formulation}

We consider a power minimization problem for secure transmission of information to the IR while maintaining the QoS of signal reception. In order to satisfy the secrecy requirements, conventional power minimization problem is formulated as

$$
\begin{aligned}
\mathbf{P 0}: \min _{\mathbf{b}_{\mathrm{d}},\left\{\mathbf{b}_{\mathrm{n}, i\}}\right.} & \left\|\mathbf{b}_{\mathrm{d}}\right\|^{2}+\sum_{i=1}^{N}\left\|\mathbf{b}_{\mathrm{n}, i}\right\|^{2} \\
\text { s.t. } & \frac{\left|\mathbf{h}_{\mathrm{d}}^{T} \mathbf{b}_{\mathrm{d}}\right|^{2}}{\sum_{i=1}^{N}\left|\mathbf{h}_{\mathrm{d}}^{T} \mathbf{b}_{\mathrm{n}, i}\right|^{2}+\sigma_{\mathrm{d}}^{2}} \geq \Gamma_{\mathrm{d}}, \\
& \frac{\left|\mathbf{h}_{\mathrm{e}, k}^{T} \mathbf{b}_{\mathrm{d}}\right|^{2}}{\sum_{i=1}^{N}\left|\mathbf{h}_{\mathrm{e}, k}^{T} \mathbf{b}_{\mathrm{n}, i}\right|^{2}+\sigma_{\mathrm{e}}^{2}} \leq \Gamma_{\mathrm{e}, k}, \forall k .
\end{aligned}
$$

The power minimization problem has been solved considering various system configurations [7], [8]. Although the solutions proposed in [7], [8] are optimal from stochastic viewpoint, the hidden power in the AN signals has been treated as harmful for the desired information, and hence, either nullified or suppressed. In the following section, we endeavour to develop precoding schemes exploiting the AN power constructively for the desired signal at the IR.

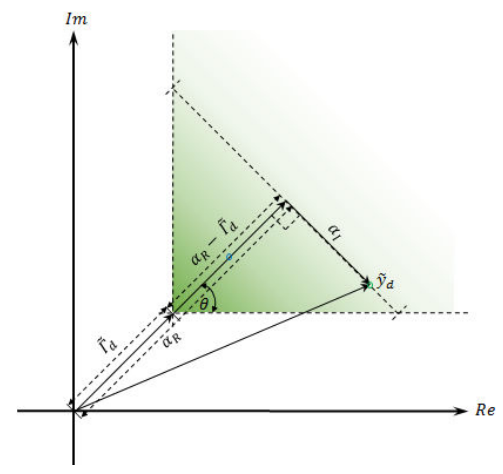

Fig. 1: Constructive AN design for the legitimate receiver.

\section{Constructive AN-BAsed Secure Precoding}

In this section, we take the above approach one step forward, by actively exploiting interference (AN in this case) constructively for the IR to reduce the required power for a given SNR threshold, while guaranteeing the secrecy constraint for the Eves. The theory and characterization criteria for constructive interference have been extensively studied in [2]-[4]. The AN signal will be constructive to the received signal at the IR if that moves the receives symbols away from the decision thresholds of the constellation (e.g., real and imaginary axes for QPSK symbols in Fig. 1). Hence we intend to keep the angle of that part aligned with the angle of the corresponding desired symbol $s_{\mathrm{d}}$ by appropriately designing the transmit beamforming vectors. We can do so by pushing the decision symbols towards the constructive regions of the modulation constellation, denoted by the green shaded areas (cf. Fig. 1).

Exploiting this concept, it can be shown that the receive SINR (4) at the IR can be rewritten as [4]

$$
\gamma_{\mathrm{d}}=\frac{\left|\mathbf{h}_{\mathrm{d}}^{T} \mathbf{b}_{\mathrm{d}} s_{\mathrm{d}}+\mathbf{h}_{\mathrm{d}}^{T} \sum_{i=1}^{N} \mathbf{b}_{\mathrm{n}, i} s_{\mathrm{n}, i}\right|^{2}}{\sigma_{\mathrm{d}}^{2}} .
$$

However, the SINR at the $k$ th Eve remains the same as in (5) since no AN signal has been made constructive to the Eves.

Thus exploiting AN power constructively, the SINR constraint (8b) can be reformulated as the following system of constraints

$$
\begin{aligned}
& \angle\left(\mathbf{h}_{\mathrm{d}}^{T} \mathbf{b}_{\mathrm{d}} s_{\mathrm{d}}+\sum_{i=1}^{N} \mathbf{h}_{\mathrm{d}}^{T} \mathbf{b}_{\mathrm{n}, i} s_{\mathrm{n}, i}\right)=\angle\left(s_{\mathrm{d}}\right) \\
& \frac{\Re\left\{\mathbf{h}_{\mathrm{d}}^{T}\left(\mathbf{b}_{\mathrm{d}}+\sum_{i=1}^{N} \mathbf{b}_{\mathrm{n}, i} e^{j\left(\phi_{\mathrm{n}, i}-\phi_{\mathrm{d}}\right)}\right)\right\}^{2}}{\sigma_{\mathrm{d}}^{2}} \geq \Gamma_{\mathrm{d}},
\end{aligned}
$$

where $\Re\{x\}$ indicates the real part of the complex number $x$ and $\angle x$ denotes the corresponding angle. Note that the phases of the AN signals in (10b) has been shifted by the phase of the desired symbol $s_{\mathrm{d}}$.

Let us denote $\tilde{y}_{\mathrm{d}} \triangleq \mathbf{h}_{\mathrm{d}}^{T}\left(\mathbf{b}_{\mathrm{d}}+\sum_{i=1}^{N} \mathbf{b}_{\mathrm{n}, i} e^{j\left(\phi_{\mathrm{n}, i}-\phi_{\mathrm{d}}\right)}\right)$ as the received signal ignoring the AWGN at the IR, with constructive AN injected, and $\alpha_{\mathrm{R}}$ and $\alpha_{\mathrm{I}}$ as the abscissa and the ordinate of the phase-adjusted signal $\tilde{y}_{\mathrm{d}}$, respectively. 
Applying basic geometric principles, it can be observed from Fig. 1 that the AN contaminated received signal $\tilde{y}_{\mathrm{d}}$ does not necessarily need to strictly align the angle of the desired signal. That is, $\tilde{y}_{\mathrm{d}}$ lays on the constructive zone of the desired symbol $s_{\mathrm{d}}$ as long as the following condition is satisfied

$$
-\theta \leq \phi_{\mathrm{d}} \leq \theta, \quad \text { i.e., } \quad \frac{\left|\alpha_{\mathrm{I}}\right|}{\alpha_{\mathrm{R}}-\tilde{\Gamma}_{\mathrm{d}}} \leq \tan \theta,
$$

where $\tilde{\Gamma}_{\mathrm{d}} \triangleq \sigma_{\mathrm{d}} \sqrt{\Gamma_{\mathrm{d}}}$ and $\theta=\pi / M, M$ is the constellation size. Thus the strict angle constraint (10a) can be relaxed as [12]

$$
\begin{aligned}
&\left|\Im\left\{\mathbf{h}_{\mathrm{d}}^{T}\left(\mathbf{b}_{\mathrm{d}}+\sum_{i=1}^{N} \mathbf{b}_{\mathrm{n}, i} e^{j\left(\phi_{\mathrm{n}, i}-\phi_{\mathrm{d}}\right)}\right)\right\}\right| \\
& \leq\left\{\Re \mathbf{h}_{\mathrm{d}}^{T}\left(\mathbf{b}_{\mathrm{d}}+\sum_{i=1}^{N} \mathbf{b}_{\mathrm{n}, i} e^{j\left(\phi_{\mathrm{n}, i}-\phi_{\mathrm{d}}\right)}\right)\right\}\left.-\sigma_{\mathrm{d}} \sqrt{\Gamma_{\mathrm{d}}}\right) \\
& \times \tan \theta
\end{aligned}
$$

where $\Im\{x\}$ indicates the imaginary part of the complex number $x$. Thus the constructive interference based secure transmit precoding optimization problem is formulated as

$$
\begin{aligned}
\mathbf{P 1 :} \min _{\mathbf{b}_{\mathrm{d}},\left\{\mathbf{b}_{\mathrm{n}, i}\right\}} & \left\|\mathbf{b}_{\mathrm{d}}+\sum_{i=1}^{N} \mathbf{b}_{\mathrm{n}, i} e^{j\left(\phi_{\mathrm{n}, i}-\phi_{\mathrm{d}}\right)}\right\|^{2} \\
\text { s.t. } & \left|\Im\left\{\mathbf{h}_{\mathrm{d}}^{T}\left(\mathbf{b}_{\mathrm{d}}+\sum_{i=1}^{N} \mathbf{b}_{\mathrm{n}, i} e^{j\left(\phi_{\mathrm{n}, i}-\phi_{\mathrm{d}}\right)}\right)\right\}\right| \\
& \leq\left(\Re\left\{\mathbf{h}_{\mathrm{d}}^{T}\left(\mathbf{b}_{\mathrm{d}}+\sum_{i=1}^{N} \mathbf{b}_{\mathrm{n}, i} e^{j\left(\phi_{\mathrm{n}, i}-\phi_{\mathrm{d}}\right)}\right)\right\}\right. \\
& \frac{\left|\mathbf{h}_{\mathrm{e}, k}^{T} \mathbf{b}_{\mathrm{d}}\right|^{2}}{\sum_{i=1}^{N}\left|\mathbf{h}_{\mathrm{e}, k}^{T} \mathbf{b}_{\mathrm{n}, i}\right|^{2}+\sigma_{\mathrm{e}}^{2}} \leq \Gamma_{\mathrm{e}, k}, \forall k .
\end{aligned}
$$

The problem (13) can be reformulated as a standard SOCP, which can be optimally solved using optimization toolboxes, e.g., CVX [13].

\section{Robust Constructive Interference Precoding}

In the previous sections, it was assumed that perfect CSI of all the nodes is available at the transmitter. However, that is a very strict assumption for many practical wireless communication systems. In particular, obtaining the perfect Eves' CSI is always a challenging task. Hence in this section, we study robust AN precoding design for scenarios when the available CSI is imperfect.

We model the imperfect CSI considering the widely used Gaussian channel error model such that the channel error vectors have circularly symmetric complex Gaussian (CSCG) distribution. Thus, the actual channels between the BS and the IR can be modeled as

$$
\mathbf{h}_{\mathrm{d}}=\hat{\mathbf{h}}_{\mathrm{d}}+\mathbf{e}_{\mathrm{d}}
$$

and that between the BS and the $k$ th Eve can be modelled as

$$
\mathbf{h}_{\mathrm{e}, k}=\hat{\mathbf{h}}_{\mathrm{e}, k}+\mathbf{e}_{\mathrm{e}, k}, \forall k,
$$

where $\hat{\mathbf{h}}_{\mathrm{d}}$ and $\hat{\mathbf{h}}_{\mathrm{e}, k}, \forall k$, denote the imperfect estimated CSI available at the $\mathrm{BS}$ and $\mathbf{e}_{\mathrm{d}}, \mathbf{e}_{\mathrm{e}, k} \in \mathbb{C}^{N_{\mathrm{T}} \times 1}, \forall k$, represent the channel uncertainties such that $\left\|\mathbf{e}_{\mathrm{d}}\right\|^{2} \leq \varepsilon_{\mathrm{d}}^{2}$, and $\left\|\mathbf{e}_{\mathrm{e}, k}\right\|^{2} \leq$ $\varepsilon_{\mathrm{e}}^{2}, \forall k$, respectively.

\section{A. Conventional AN-Aided Robust Secure Precoding}

Conventional AN-aided downlink robust secrecy power minimization problem with SINR constraints is formulated as

$$
\begin{aligned}
& \min _{\mathbf{b}_{\mathrm{d}},\left\{\mathbf{b}_{\mathrm{n}, i}\right\}}\left\|\mathbf{b}_{\mathrm{d}}\right\|^{2}+\sum_{i=1}^{N}\left\|\mathbf{b}_{\mathrm{n}, i}\right\|^{2} \\
& \text { s.t. } \min _{\left\|\mathbf{e}_{\mathrm{d}}\right\| \leq \varepsilon_{\mathrm{d}}} \frac{\left|\mathbf{h}_{\mathrm{d}}^{T} \mathbf{b}_{\mathrm{d}}\right|^{2}}{\sum_{i=1}^{N}\left|\mathbf{h}_{\mathrm{d}}^{T} \mathbf{b}_{\mathrm{n}, i}\right|^{2}+\sigma_{\mathrm{d}}^{2}} \geq \Gamma_{\mathrm{d}}, \quad \text { (16b) } \\
& \max _{\left\|\mathbf{e}_{\mathrm{e}, k}\right\| \leq \varepsilon_{\mathrm{e}}} \frac{\left|\mathbf{h}_{\mathrm{e}, k}^{T} \mathbf{b}_{\mathrm{d}}\right|^{2}}{\sum_{i=1}^{N}\left|\mathbf{h}_{\mathrm{e}, k}^{T} \mathbf{b}_{\mathrm{n}, i}\right|^{2}+\sigma_{\mathrm{e}}^{2}} \leq \Gamma_{\mathrm{e}, k} .(16 \mathrm{c})
\end{aligned}
$$

Due to the spherical channel uncertainty model, constraints (16b) and (16c) actually involve infinitely many constraints which makes the problem (16) very difficult to solve. However, applying $\mathcal{S}$-procedure [8, Lemma 2], the inequality constraints in (16) can be transformed into convex linear matrix inequality constraints and thus problem (16) can be readily solved using existing solvers.

\section{B. Constructive AN-Aided Robust Secure Precoding}

With the deterministic channel uncertainty model described above, we consider worst-case based robust design. Thus the constructive AN based robust power minimization problem can be formulated as

$$
\begin{aligned}
\min _{\mathbf{b}_{\mathrm{d}},\left\{\overline{\mathbf{b}}_{\mathrm{n}, i}\right\}} & \left\|\mathbf{b}_{\mathrm{d}}+\sum_{i=1}^{N} \overline{\mathbf{b}}_{\mathrm{n}, i}\right\|^{2} \\
\text { s.t. } & \left|\Im\left\{\mathbf{h}_{\mathrm{d}}^{T}\left(\mathbf{b}_{\mathrm{d}}+\sum_{i=1}^{N} \overline{\mathbf{b}}_{\mathrm{n}, i}\right)\right\}\right| \\
& \leq\left(\Re\left\{\mathbf{h}_{\mathrm{d}}^{T}\left(\mathbf{b}_{\mathrm{d}}+\sum_{i=1}^{N} \overline{\mathbf{b}}_{\mathrm{n}, i}\right)\right\}-\sigma_{\mathrm{d}} \sqrt{\Gamma_{\mathrm{d}}}\right) \tan \theta \\
& \frac{\left|\mathbf{h}_{\mathrm{e}, k}^{T} \mathbf{b}_{\mathrm{d}}\right|^{2}}{\sum_{i=1}^{N}\left|\mathbf{h}_{\mathrm{e}, k}^{T} \overline{\mathbf{b}}_{\mathrm{n}, i}\right|^{2}+\sigma_{\mathrm{e}}^{2}} \leq \varepsilon_{\mathrm{d}},
\end{aligned}
$$

where $\overline{\mathbf{b}}_{\mathrm{n}, i} \triangleq \mathbf{b}_{\mathrm{n}, i} e^{j\left(\phi_{\mathrm{n}, i}-\phi_{\mathrm{d}}\right)}$. Considering the real and imaginary parts of each complex vector separately, we have

$$
\begin{aligned}
\mathbf{h}_{\mathrm{d}} & =\hat{\mathbf{h}}_{R, \mathrm{~d}}+j \hat{\mathbf{h}}_{I, \mathrm{~d}}+\mathbf{e}_{R, \mathrm{~d}}+j \mathbf{e}_{I, \mathrm{~d}}, \\
\mathbf{b}_{\mathrm{d}} & =\mathbf{b}_{\mathrm{d} R}+j \mathbf{b}_{\mathrm{d} I}, \\
\mathbf{b}_{\mathrm{n}, i} & =\mathbf{b}_{\mathrm{n} R, i}+j \mathbf{b}_{\mathrm{n} I, i}, \forall i,
\end{aligned}
$$


where the subscripts $R$ and $I$ indicate the real and imaginary components of the corresponding complex notation, respectively. As such, we have the real part,

$$
\begin{aligned}
\Re\left\{\mathbf{h}_{\mathrm{d}}^{T}\left(\mathbf{b}_{\mathrm{d}}+\sum_{i=1}^{N} \overline{\mathbf{b}}_{\mathrm{n}, i}\right)\right\} & =\tilde{\mathbf{h}}_{\mathrm{d}}^{T} \mathbf{b}_{\mathrm{d}, 1}+\sum_{i=1}^{N} \tilde{\mathbf{h}}_{\mathrm{d}}^{T} \tilde{\mathbf{b}}_{\mathrm{n} 1, i} \\
& +\tilde{\mathbf{e}}_{\mathrm{d}}^{T} \mathbf{b}_{\mathrm{d}, 1}+\sum_{i=1}^{N} \tilde{\mathbf{e}}_{\mathrm{d}}^{T} \tilde{\mathbf{b}}_{\mathrm{n} 1, i},
\end{aligned}
$$

where $\tilde{\mathbf{h}}_{\mathrm{d}} \triangleq\left[\begin{array}{cc}\hat{\mathbf{h}}_{R, \mathrm{~d}}^{T} & \hat{\mathbf{h}}_{I, \mathrm{~d}}^{T}\end{array}\right]^{T}, \tilde{\mathbf{e}}_{\mathrm{d}} \triangleq\left[\begin{array}{cc}\mathbf{e}_{R, \mathrm{~d}}^{T} & \mathbf{e}_{I, \mathrm{~d}}^{T}\end{array}\right]^{T}, \mathbf{b}_{\mathrm{d}, 1} \triangleq$ $\left[\begin{array}{ll}\mathbf{b}_{R}^{T} & -\mathbf{b}_{I}^{T}\end{array}\right]^{T}$, and $\tilde{\mathbf{b}}_{\mathrm{n} 1, i} \triangleq\left[\begin{array}{ll}\mathbf{b}_{\mathrm{n} R, i}^{T} & -\mathbf{b}_{\mathrm{n} I, i}^{T}\end{array}\right]^{T}, \forall i$. On the other hand, the imaginary component can be expressed as

$$
\begin{aligned}
\Im\left\{\mathbf{h}_{\mathrm{d}}^{T}\left(\mathbf{b}_{\mathrm{d}}+\sum_{i=1}^{N} \overline{\mathbf{b}}_{\mathrm{n}, i}\right)\right\} & =\tilde{\mathbf{h}}_{\mathrm{d}}^{T} \mathbf{b}_{\mathrm{d}, 2}+\sum_{i=1}^{N} \tilde{\mathbf{h}}_{\mathrm{d}}^{T} \tilde{\mathbf{b}}_{\mathrm{n} 2, i} \\
& +\tilde{\mathbf{e}}_{\mathrm{d}}^{T} \mathbf{b}_{\mathrm{d}, 2}+\sum_{i=1}^{N} \tilde{\mathbf{e}}_{\mathrm{d}}^{T} \tilde{\mathbf{b}}_{\mathrm{n} 2, i},
\end{aligned}
$$

with $\mathbf{b}_{\mathrm{d}, 2} \triangleq\left[\begin{array}{ll}\mathbf{b}_{I}^{T} & \mathbf{b}_{R}^{T}\end{array}\right]^{T}$, and $\tilde{\mathbf{b}}_{\mathrm{n} 2, i} \triangleq\left[\begin{array}{ll}\mathbf{b}_{\mathrm{n} I, i}^{T} & \mathbf{b}_{\mathrm{n} R, i}^{T}\end{array}\right]^{T}, \forall i$. Thus the constraint (17b) can be explicitly expressed as the following two constraints

$$
\begin{gathered}
\max _{\left\|\mathbf{e}_{\mathrm{d}}\right\| \leq \varepsilon_{\mathrm{d}}} \tilde{\mathbf{h}}_{\mathrm{d}}^{T} \mathbf{b}_{\mathrm{d}, 2}+\sum_{i=1}^{N} \tilde{\mathbf{h}}_{\mathrm{d}}^{T} \tilde{\mathbf{b}}_{\mathrm{n} 2, i}+\tilde{\mathbf{e}}_{\mathrm{d}}^{T} \mathbf{b}_{\mathrm{d}, 2}+\sum_{i=1}^{N} \tilde{\mathbf{e}}_{\mathrm{d}}^{T} \tilde{\mathbf{b}}_{\mathrm{n} 2, i} \\
-\left(\tilde{\mathbf{h}}_{\mathrm{d}}^{T} \mathbf{b}_{\mathrm{d}, 1}+\sum_{i=1}^{N} \tilde{\mathbf{h}}_{\mathrm{d}}^{T} \tilde{\mathbf{b}}_{\mathrm{n} 1, i}+\tilde{\mathbf{e}}_{\mathrm{d}}^{T} \mathbf{b}_{\mathrm{d}, 1}+\sum_{i=1}^{N} \tilde{\mathbf{e}}_{\mathrm{d}}^{T} \tilde{\mathbf{b}}_{\mathrm{n} 1, i}\right) \tan \theta \\
\quad+\sigma_{\mathrm{d}} \sqrt{\Gamma_{\mathrm{d}}} \tan \theta \leq 0 \\
\max _{\left\|\mathbf{e}_{\mathrm{d}}\right\| \leq \varepsilon_{\mathrm{d}}}-\left(\tilde{\mathbf{h}}_{\mathrm{d}}^{T} \mathbf{b}_{\mathrm{d}, 2}+\sum_{i=1}^{N} \tilde{\mathbf{h}}_{\mathrm{d}}^{T} \tilde{\mathbf{b}}_{\mathrm{n} 2, i}+\tilde{\mathbf{e}}_{\mathrm{d}}^{T} \mathbf{b}_{\mathrm{d}, 2}+\sum_{i=1}^{N} \tilde{\mathbf{e}}_{\mathrm{d}}^{T} \tilde{\mathbf{b}}_{\mathrm{n} 2, i}\right) \\
-\left(\tilde{\mathbf{h}}_{\mathrm{d}}^{T} \mathbf{b}_{\mathrm{d}, 1}+\sum_{i=1}^{N} \tilde{\mathbf{h}}_{\mathrm{d}}^{T} \tilde{\mathbf{b}}_{\mathrm{n} 1, i}+\tilde{\mathbf{e}}_{\mathrm{d}}^{T} \mathbf{b}_{\mathrm{d}, 1}+\sum_{i=1}^{N} \tilde{\mathbf{e}}_{\mathrm{d}}^{T} \tilde{\mathbf{b}}_{\mathrm{n} 1, i}\right) \tan \theta \\
+\sigma_{\mathrm{d}} \sqrt{\Gamma_{\mathrm{d}}} \tan \theta \leq 0 .
\end{gathered}
$$

Now, the constraint $(17 \mathrm{c})$ can be rewritten as

$$
\max _{\left\|\mathbf{e}_{\mathrm{e}, k}\right\| \leq \varepsilon_{\mathrm{e}}}\left\|\left[\tilde{\mathbf{h}}_{\mathrm{e}, k}^{T} \overline{\mathbf{B}}_{\mathrm{n}}, \quad \sigma_{\mathrm{e}}\right]\right\| \geq \frac{1}{\sqrt{\Gamma_{\mathrm{e}, k}}} \tilde{\mathbf{h}}_{\mathrm{e}, k}^{T} \mathbf{b}_{\mathrm{d}, 1}, \forall k,
$$

where $\tilde{\mathbf{h}}_{\mathrm{e}, k} \triangleq\left[\begin{array}{ll}\mathbf{h}_{\mathrm{e} R, k}^{T} & \mathbf{h}_{\mathrm{e} I, k}^{T}\end{array}\right]^{T}$ and $\mathbf{B}_{\mathrm{n}}$ is defined as $\overline{\mathbf{B}}_{\mathrm{n}} \triangleq$ $\left[\begin{array}{cc}\Re\left\{\mathbf{B}_{\mathrm{n}}\right\} & \Im\left\{\mathbf{B}_{\mathrm{n}}\right\} \\ \Im\left\{\mathbf{B}_{\mathrm{n}}\right\} & \Re\left\{\mathbf{B}_{\mathrm{n}}\right\}\end{array}\right]$ with $\mathbf{B}_{\mathrm{n}} \triangleq\left[\mathbf{b}_{\mathrm{n}, 1}, \cdots, \mathbf{b}_{\mathrm{n}, N}\right]$. Applying $S$-procedure [14], the SOC constraint (25) can be rewritten as an equivalent linear matrix inequality (LMI)

$$
\begin{aligned}
& f_{k}\left(\overline{\mathbf{B}}_{\mathrm{n}}, \tilde{\mathbf{h}}_{\mathrm{e}, k}\right) \triangleq
\end{aligned}
$$

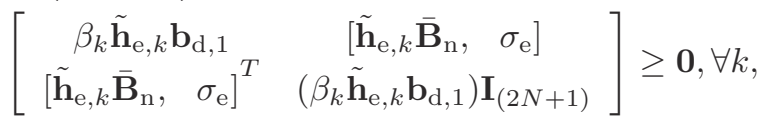

where $\beta_{k} \triangleq \frac{1}{\sqrt{\Gamma_{\mathrm{e}, k}}}$. By substituting $\mathbf{h}_{\mathrm{e}, k}=\hat{\mathbf{h}}_{\mathrm{e}, k}+\mathbf{e}_{\mathrm{e}, k}$ in the LMI above, we obtain

$$
\bar{f}_{k}\left(\overline{\mathbf{B}}_{\mathrm{n}}, \tilde{\mathbf{h}}_{\mathrm{e}, k}\right) \triangleq f_{k}\left(\overline{\mathbf{B}}_{\mathrm{n}}, \tilde{\mathbf{h}}_{\mathrm{e}, k}\right)+\boldsymbol{\Psi}_{k} \boldsymbol{\Lambda}_{k}+\boldsymbol{\Lambda}_{k}^{T} \boldsymbol{\Psi}_{k}^{T},
$$

where $\boldsymbol{\Psi}_{k} \triangleq \mathbf{I}_{2 N+2} \otimes \tilde{\mathbf{e}}_{\mathrm{e}, k}$ and $\boldsymbol{\Lambda}_{k} \triangleq$ $\left[\begin{array}{cc}\frac{1}{2} \beta_{k} \mathbf{b}_{\mathrm{d}, 1} & {\left[\begin{array}{c}\overline{\mathbf{B}}_{\mathrm{n}}, \mathbf{0}\end{array}\right]} \\ \mathbf{0} & \left(\frac{1}{2} \beta_{k}\right) \mathbf{I}_{(2 N+1)} \otimes \mathbf{b}_{\mathrm{d}, 1}\end{array}\right]$. Note that the uncertainty matrix $\boldsymbol{\Psi}_{k}$ belongs to a subspace of block diagonal matrices with equal blocks. Hence, the spectral norm of $\boldsymbol{\Psi}_{k}$ is $\left\|\boldsymbol{\Psi}_{k}\right\|=\left\|\tilde{\mathbf{e}}_{\mathrm{e}, k}\right\| \leq \varepsilon_{\mathrm{e}}$.

Now, (27) holds if and only if there exists a $\mu_{k}$ such that

$$
\left[\begin{array}{cc}
f_{k}\left(\overline{\mathbf{B}}_{\mathrm{n}}, \tilde{\mathbf{h}}_{\mathrm{e}, k}\right)-\mu_{k} \mathbf{I}_{(2 N+1)} & \boldsymbol{\Lambda}_{k}^{T} \\
\boldsymbol{\Lambda}_{k} & \mu_{k} \varepsilon_{\mathrm{e}}^{-2} \mathbf{I}_{q}
\end{array}\right] \succeq \mathbf{0}, \forall k,
$$

where $q=4 N_{\mathrm{T}}(N+1)$. By replacing the CSI error bounds in the constraints (23) and (24), the robust problem (17) can thus be reformulated as

$$
\begin{aligned}
& \min _{\mathbf{b}_{1}, \mathbf{b}_{2}}\left\|\mathbf{b}_{2}\right\|^{2} \text { s.t. } \\
& \tilde{\mathbf{h}}_{\mathrm{d}}^{T} \mathbf{b}_{\mathrm{d}, 2}+\sum_{i=1}^{N} \tilde{\mathbf{h}}_{\mathrm{d}}^{T} \tilde{\mathbf{b}}_{\mathrm{n} 2, i}-\left(\tilde{\mathbf{h}}_{\mathrm{d}}^{T} \mathbf{b}_{\mathrm{d}, 1}+\sum_{i=1}^{N} \tilde{\mathbf{h}}_{\mathrm{d}}^{T} \tilde{\mathbf{b}}_{\mathrm{n} 1, i}\right) \tan \theta \\
& +\varepsilon_{\mathrm{d}}\left\|\mathbf{b}_{\mathrm{d}, 2}+\sum_{i=1}^{N} \tilde{\mathbf{b}}_{\mathrm{n} 2, i}-\left(\mathbf{b}_{\mathrm{d}, 1}+\sum_{i=1}^{N} \tilde{\mathbf{b}}_{\mathrm{n} 1, i}\right) \tan \theta\right\| \\
& +\sigma_{\mathrm{d}} \sqrt{\Gamma_{\mathrm{d}}} \tan \theta \leq 0, \\
& -\left(\tilde{\mathbf{h}}_{\mathrm{d}}^{T} \mathbf{b}_{\mathrm{d}, 2}+\sum_{i=1}^{N} \tilde{\mathbf{h}}_{\mathrm{d}}^{T} \tilde{\mathbf{b}}_{\mathrm{n} 2, i}+\left(\tilde{\mathbf{h}}_{\mathrm{d}}^{T} \mathbf{b}_{\mathrm{d}, 1}+\sum_{i=1}^{N} \tilde{\mathbf{h}}_{\mathrm{d}}^{T} \tilde{\mathbf{b}}_{\mathrm{n} 1, i}\right) \tan \theta\right) \\
& -\varepsilon_{\mathrm{d}}\left\|\mathbf{b}_{\mathrm{d}, 2}+\sum_{i=1}^{N} \tilde{\mathbf{b}}_{\mathrm{n} 2, i}+\left(\mathbf{b}_{\mathrm{d}, 1}+\sum_{i=1}^{N} \tilde{\mathbf{b}}_{\mathrm{n} 1, i}\right) \tan \theta\right\| \\
& +\sigma_{\mathrm{d}} \sqrt{\Gamma_{\mathrm{d}}} \tan \theta \leq 0, \\
& {\left[\begin{array}{cc}
f_{k}\left(\overline{\mathbf{B}}_{\mathrm{n}}, \tilde{\mathbf{h}}_{\mathrm{e}, k}\right)-\mu_{k} \mathbf{I}_{(2 N+1)} & \boldsymbol{\Lambda}_{k}^{T} \\
\boldsymbol{\Lambda}_{k} & \mu_{k} \varepsilon_{\mathrm{e}}^{-2} \mathbf{I}_{q}
\end{array}\right] \succeq \mathbf{0}, \forall k .}
\end{aligned}
$$

The problem (29) can be efficiently solved using existing solvers [13].

\section{Simulation Results}

This section presents numerical simulation results to evaluate the performance of the proposed constructive interference based PLS algorithms in a MISO wiretap channel. For simplicity, it was assumed that $\Gamma_{\mathrm{e}, k}=\Gamma_{\mathrm{e}}, \forall k$ and $\sigma_{\mathrm{d}}^{2}=\sigma_{\mathrm{e}}^{2}=1$. Unless otherwise specified, $N=3$ and QPSK is the modulation scheme considered. All the estimated channel vectors are generated as independent and identically distributed complex Gaussian random variables with mean zero and the TGn path-loss model for urban cellular environment is adopted considering a path-loss exponent of 2.7. All simulation results are averaged over 1000 independent channel realizations, unless explicitly mentioned. In the following simulations, we compare the performance of the proposed approaches with that of the conventional AN-aided precoding scheme in [7] as the benchmark. Specifically, we denote the conventional precoding schemes as 'Conv Prec' and the constructive interference based precoding scheme developed in Sections IV and V-B as 'Const Prec' in the figures below. 


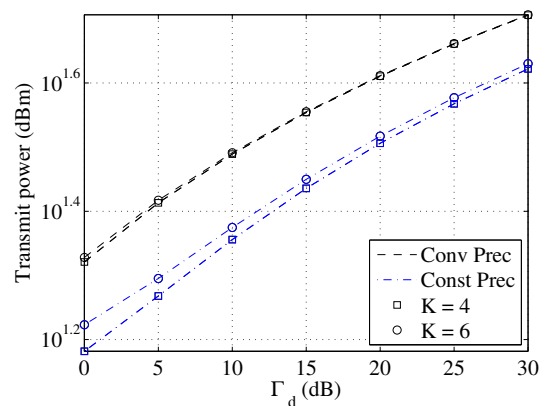

Fig. 2: Transmit power $P_{\mathrm{T}}$ versus required SINR at IR $\Gamma_{\mathrm{d}}$ with $N_{\mathrm{T}}=8, K=$ 4,6 , and $\Gamma_{\mathrm{e}}=5(\mathrm{~dB})$.

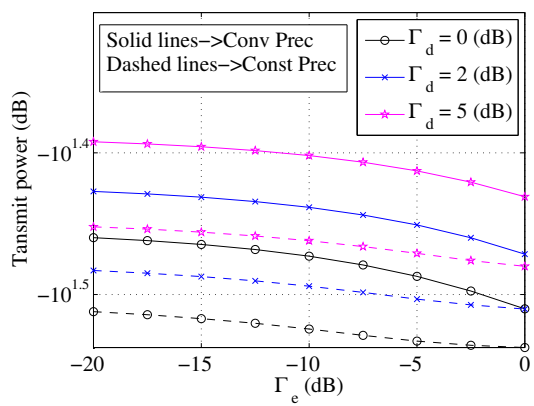

Fig. 3: Transmit power $P_{\mathrm{T}}$ versus Eves' SINR $\Gamma_{\mathrm{e}}$ with $N_{\mathrm{T}}=6, K=4$, and $\Gamma_{\mathrm{d}}=20(\mathrm{~dB})$.

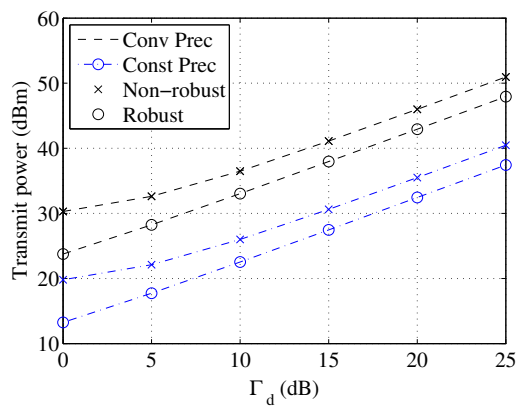

Fig. 4: Transmit power $P_{\mathrm{T}}$ versus required SINR $\Gamma_{\mathrm{d}}$ with $N_{\mathrm{T}}=6, K=3$, $\Gamma_{\mathrm{e}}=5(\mathrm{~dB})$, and $\varepsilon_{\mathrm{d}}=0.1, \varepsilon_{\mathrm{e}}=0.3$.
We start the performance analysis of the proposed precoding schemes assuming perfect CSI. Fig. 2 shows the average transmit power versus the SINR requirement at the IR required for the proposed constructive AN precoding optimization scheme (in problem (13)) as compared with the conventional AN precoding scheme (in problem (8)) for $K=4$ and 6 , $N_{\mathrm{T}}=8$ and $\Gamma_{\mathrm{e}}=5(\mathrm{~dB})$. It can be observed that the proposed constructive interference algorithms achieve significant power gains compared to the conventional AN precoding scheme.

In the next example, we examine the transmit power requirement against the maximum allowable eavesdropping SINR $\Gamma_{\mathrm{e}}$. Fig. 3 plots the average transmit power $P_{\mathrm{T}}$ versus $\Gamma_{\mathrm{e}}$ for $N_{\mathrm{T}}=6, K=4$ and different values of $\Gamma_{\mathrm{d}}$. The results in Fig. 3 are consistent with those in Fig. 2 in the sense that increased SINR threshold at the IR requires higher transmit power. However, with the increase in the allowable SINR threshold at the Eves, the required transmit power gradually decreases due to the relaxed eavesdropping constraints. Also, in any case, the constructive interference based precoding schemes outperform the conventional AN-aided secure precoding schemes.

Finally, we analyze the performance of the proposed robust beamforming design with $N_{\mathrm{T}}=6, K=3, \Gamma_{\mathrm{e}}=5(\mathrm{~dB})$, and $\varepsilon_{\mathrm{d}}=0.1, \varepsilon_{\mathrm{e}}=0.3$, when imperfect CSI is available at the BS. In Fig. 4, the robust schemes indicate the solution to the problems (16) and (29), respectively, for conventional and constructive AN based precoding schemes. On the other hand, the 'Non-robust' scheme is designed treating the imperfect channel estimates available at the BS as the perfect CSI, hence yields noticeable performance degradation. The proposed constructive interference based robust secure beamforming schemes demonstrate significant transmit power gain.

\section{CONCLUSIONS}

We proposed the novel idea of designing the AN-aided secure precoding schemes as constructive to the IR and destructive to the Eves at the same time. The concept opens up new opportunities for expanding the secrecy rate regions. We studied the downlink transmit power minimization problem considering both perfect and imperfect CSI at the BS.
Simulation results demonstrated that significant performance gain is achievable by the proposed constructive AN precoding schemes compared to the conventional schemes and have established the proposed approach as a new dimension in the design of PLS.

\section{REFERENCES}

[1] M. R. A. Khandaker and Y. Rong, "Interference MIMO relay channel: Joint power control and transceiver-relay beamforming," IEEE Trans. Signal Process., vol. 60, pp. 6509-6518, Dec. 2012.

[2] G. Zheng, I. Krikidis, C. Masouros, S. Timotheou, D. A. Toumpakaris, and Z. Ding, "Rethinking the role of interference in wireless networks," IEEE Commun. Mag., vol. 52, pp. 152-158, Nov. 2014.

[3] C. Masouros and E. Alsusa, "Dynamic linear precoding for the exploitation of known interference in MIMO broadcast systems," IEEE Trans. Wireless Commun., vol. 8, pp. 1396-1404, Mar. 2009.

[4] C. Masouros and G. Zheng, "Exploiting known interference as green signal power for downlink beamforming optimization," IEEE Trans. Signal Process., vol. 63, pp. 3628-3640, July 2015.

[5] M. Alodeh, S. Chatzinotas, and B. Ottersten, "Constructive multiuser interference in symbol level precoding for the MISO downlink channel," IEEE Trans. Signal Process., vol. 63, pp. 2239-2252, May 2015.

[6] S. Goel and R. Negi, "Guaranteeing secrecy using artificial noise," IEEE Trans. Wireless Commun., vol. 7, pp. 2180-2189, June 2008.

[7] W.-C. Liao, T.-H. Chang, W.-K. Ma, and C.-Y. Chi, "QoS-based transmit beamforming in the presence of eavesdroppers: An optimized artificialnoise-aided approach,' IEEE Trans. Signal Process., vol. 59, pp. 12021216, Mar. 2011.

[8] M. R. A. Khandaker and K.-K. Wong, "Masked beamforming in the presence of energy-harvesting eavesdroppers," IEEE Trans. Inf. Forensics and Security, vol. 10, pp. 40-54, Jan. 2015.

[9] M. R. A. Khandaker, K.-K. Wong, Y. Zhang, and Z. Zheng, "Probabilistically robust SWIPT for secrecy MISOME systems," IEEE Trans. Inf. Forensics Security, vol. 12, pp. 211-226, Jan. 2017.

[10] M. Daly and J. Bernhard, "Directional modulation technique for phased arrays," IEEE Trans. Antennas Propag., vol. 57, pp. 2633-2640, Sep. 2009.

[11] A. Kalantari, M. Soltanalian, S. Maleki, S. Chatzinotas, and B. Ottersten, "Directional modulation via symbol-level precoding: A way to enhance security," IEEE J. Sel. Topics Signal Process., available online at http://arXiv:1606.04488v2, Aug. 2016.

[12] M. R. A. Khandaker, C. Masouros, and K.-K. Wong, "Constructive interference based secure precoding: A new dimension in physical layer security," IEEE Trans. Signal Process., available online at arXiv: 1612.08465, 2017

[13] M. Grant and S. Boyd, "CVX: Matlab software for disciplined convex programming (web page and software)." http://cvxr.com/cvx, Apr., 2010.

[14] S. Boyd and L. Vandenberghe, Convex Optimization. Cambridge, U. K.: Cambridge University Press, 2004. 